\title{
Corneal Biomechanics for Ocular Hypertension, Primary Open-Angle Glaucoma, and Amyloidotic Glaucoma: A Comparative Study by Corvis ST
}

\author{
Nisa Silva (1) \\ André Ferreira (ID) ${ }^{1,2}$ \\ Pedro Manuel Baptista (D) \\ Ana Figueiredo' \\ Rita Reis' \\ Isabel Sampaio' \\ João Beirão ${ }^{1,3}$ \\ Riccardo Vinciguerra $\mathbb{D}^{4,5}$ \\ Pedro Menéres (ID) ${ }^{1,3}$ \\ Maria João Menéres (DD ${ }^{1,3}$ \\ 'Ophthalmology Department, Centro \\ Hospitalar Universitário do Porto, Porto, \\ 4099-00 I, Portugal; ' ${ }^{2}$ nit of Anatomy, \\ Department of Biomedicine, Faculty of \\ Medicine of University of Porto, Porto, \\ 4200-319, Portugal; ${ }^{3}$ Department of \\ Ophthalmology, Instituto de Ciências \\ Biomédicas Abel Salazar, University of \\ Porto, Porto, 4050-3I3, Portugal; \\ ${ }^{4}$ Ophthalmology Department, Humanitas \\ San Pio X Hospital, Milan, Italy; ${ }^{5}$ The \\ School of Engineering, University of \\ Liverpool, Liverpool, UK
}

Background: To evaluate biomechanical parameters of the cornea provided by Corvis ST in patients with ocular hypertension, primary open-angle glaucoma, and amyloidotic glaucoma and to compare with healthy controls.

Methods: This was a cross-sectional study of patients with ocular hypertension, primary open-angle glaucoma, and amyloidotic glaucoma that underwent Corvis ST imaging. Primary outcome was the comparison of corneal biomechanical parameters between study groups after adjusting for age, gender, Goldmann intraocular pressure (GAT-IOP), and prostaglandin analogues medication. Secondary outcome was the comparison of different IOP measurements in each group.

Results: One hundred and eighty-three eyes from 115 patients were included: 61 with primary open-angle glaucoma, 32 with amyloidotic glaucoma, 37 with ocular hypertension and 53 were healthy controls. Amyloidotic glaucoma group had smaller radius $(p=0.025)$, lower deflection amplitude at highest concavity $(\mathrm{p}=0.019)$, and higher integrated radius $(p=0.014)$ than controls. Ocular hypertension group had higher stiffness parameter at first applanation $(\mathrm{p}=0.043)$ than those with primary open-angle glaucoma, and higher stressstrain index $(p=0.049)$ than those with amyloidotic glaucoma. Biomechanically corrected intraocular pressure was significantly lower than Goldmann intraocular pressure in group with primary open-angle glaucoma $(\mathrm{p}=0.005)$ and control group $(\mathrm{p}=0.013)$, and Goldmann intraocular pressure adjusted for pachymetry in group with primary open-angle glaucoma $(\mathrm{p}=0.01)$.

Conclusion: Eyes with amyloidotic glaucoma have more deformable corneas, while eyes with ocular hypertension have less deformable corneas. These findings may be linked to the susceptibility to glaucomatous damage and progression. There were significant differences between Goldmann applanation tonometry and biomechanically corrected intraocular ocular pressure provided by Corvis ST.

Keywords: corneal biomechanics, ocular hypertension, glaucoma, amyloidosis, Goldmann tonometry

\section{Introduction}

Corneal biomechanics has gained particular interest when the Ocular Response Analyzer (ORA; Reichert Ophthalmic Instruments, Depew, New York) was launched in the early $20^{\text {th }}$ century. ${ }^{1}$ Later, Corvis ST (Corneal Visualization Scheimpflug Technology, Oculus; Wetzlar, Germany) was introduced. Both devices are noncontact tonometers that use an air puff to applanate the cornea. Corvis ST performs a comprehensive and detailed evaluation of the cornea
Correspondence: Nisa Silva Ophthalmology Department, Centro Hospitalar Universitário do Porto, Portugal Largo do Prof. Abel Salazar, Porto, 4099-00I, Portugal

Tel +35191836766I

Fax +35I 222077500

Email nisapinhosilva@gmail.com 
through cross-sectional images captured by a high-speed Scheimpflug camera during the deformation cycle, providing more data than ORA.

In glaucoma, corneal biomechanics may contribute to elucidate the pathophysiologic mechanisms leading to axonal degeneration that are still unclear and to optimize the accuracy of intraocular pressure (IOP), the only proved modifiable risk factor for glaucoma onset and progression. $^{2}$

It has been speculated that biomechanical properties of peripapillary sclera influence the damage to the optic nerve head $(\mathrm{ONH})$ in response to variations of IOP. ${ }^{3}$ An excessive pressure-induced deformation of the lamina cribrosa (LC) eventually triggers the dysfunction and death of the retinal ganglion cell axons, and it may be exacerbated when the peripapillary sclera is more deformable. Currently, it is challenging to assess the biomechanical behavior of the posterior sclera and LC in vivo. The cornea shares a similar constitution on collagen fibrils and proteoglycan-rich extrafibrillar matrix with sclera. ${ }^{4}$ Therefore, corneal biomechanics has been regarded as an indirect measure of scleral and LC elasticity, and some studies have found correlations between corneal biomechanical parameters and the indexes of glaucoma progression. ${ }^{5,6}$ The biomechanical properties of the cornea have been investigated with the aim of helping to characterize the individual susceptibility to glaucomatous damage.

Currently, all medical and surgical treatments aim to lower IOP. Several types of tonometers emerged to minimize the influence of corneal factors on IOP, but Goldmann applanation tonometry (GAT) remains the gold standard. GAT estimates the amount of force that is required to flatten an area of the cornea with a diameter of $3.06 \mathrm{~mm} .^{7}$ As such, the accuracy of IOP measured by GAT (GAT-IOP) is affected by corneal characteristics. Central corneal thickness (CCT) was the first parameter shown to influence the IOP measurement. ${ }^{8}$ Nowadays, beyond pachymetry, other corneal biomechanical parameters can be measured using ORA or Corvis ST and an IOP corrected for corneal biomechanics is further provided by both devices. To date, it has not yet been elucidated whether the biomechanically corrected IOP (bIOP) is more accurate than GAT-IOP and which is the role of bIOP in clinical practice.

In this study, we evaluated the biomechanical parameters of the cornea provided by Corvis ST in patients with primary open-angle glaucoma (POAG), amyloidotic glaucoma (AG), and ocular hypertension (OHT), and compared with healthy controls.

\section{Methods}

This was an observational cross-sectional study. Patients were recruited between July and September 2020 from ophthalmology outpatient clinic of a tertiary center, Centro Hospitalar Universitário do Porto. The research adhered to the principles of the Declaration of Helsinki and its latest amendment (Brazil, 2013), and complied with the requirements of the institute's committee on human research.

To be included in this study, the diagnosis made by a glaucoma senior specialist (A.FIG, I.S., R.R. and M.J. M.) had to match one of the group's definition. Healthy controls had an untreated IOP lower than $21 \mathrm{mmHg}$, normal optic discs in fundoscopy, and no other ocular disorders (except for senile cataract). POAG and OHT were defined according to European Glaucoma Guidelines, $4^{\text {th }}$ edition. ${ }^{8}$ AG was classified as a secondary open-angle glaucoma affecting patients with the genetic diagnosis of hereditary transthyretin-related amyloidosis (ATTR), and amyloid deposition along the pupil border in the slit-lamp examination.

The exclusion criteria included age below 40 years, high refractive errors (spherical refraction $>6$ diopters and cylinder correction $>3$ diopters), corneal disease, contact lens wear, shallow anterior chamber (Van Herick grading <3), uncontrolled GAT-IOP $(>21 \mathrm{mmHg})$, previous glaucoma or refractive surgery, any ophthalmic surgery in the last 6 months, and a quality score (QS) other than "OK" in Corvis ST.

A comprehensive ophthalmologic examination was performed including a review of medical history, bestcorrected visual acuity (BCVA) evaluation, slit-lamp biomicroscopy, GAT, fundoscopy, and Corvis ST, in the same schedule. GAT-IOP was corrected for CCT (GAT-IOPadj) based on the validated Ehler's correction algorithm. ${ }^{9}$ BCVA registered in decimals was converted to the logarithm of the minimum angle of resolution (LogMAR) equivalent. $^{10}$

Corvis ST (software version 1.6r2015) is a noncontact tonometer coupled with a high-speed Scheimpflugcamera (4330 frames/sec) to record the movements of the cornea in response to an air puff, which are then displayed on the built-in control panel in ultra-slow motion. The air puff forces the cornea through distinct phases: an ingoing phase in which the cornea passes from 
its resting shape through a first applanation (A1) into a concave shape (highest concavity, HC); an outgoing phase, which features a second point of applanation (A2) prior to the cornea returning to its normal resting state. Several biomechanical parameters are recorded during the deformation cycle. For the three main points (A1, $\mathrm{HC}$, and A2), Corvis ST calculates the time, velocity, deformation and deflection amplitudes, and area and length of deflection. At HC, peak distance (PD) and radius ( $\mathrm{Rad}$ ) are additionally measured. Mathematically derived parameters, such as deformation amplitude ratio (DA ratio), integrated radius $(1 / \mathrm{R})$, stiffness parameter at A1 (SPA1), and stress-strain index (SSI), were recently integrated into the data output of Corvis ST. A more deformable cornea is characterized by lower time (A1T), smaller deflection length (A1DeflL) and higher velocity (A1V), at $\mathrm{A} 1$; higher deformation (HCDA) and deflection amplitudes (HCDeflA), and smaller PD and $\mathrm{Rad}$, at $\mathrm{HC}$; smaller deflection length (A2DeflL) and lower velocity (A2V), at $\mathrm{A} 2$; higher DA ratio and 1/R; lower SPA1 and SSI. ${ }^{11-13}$ The opposite is found in stiffer and less deformable corneas. Furthermore, bIOP and CCT (with good reproducibility results) ${ }^{14,15}$ are provided by the device.

Primary outcome was the comparison of corneal biomechanical parameters provided by Corvis ST between study groups. Secondary outcome was the comparison of different IOP measurements, particularly GAT-IOP, GATIOPadj, and bIOP, in each group.

All statistical analyses were performed using IBM SPSS $^{\circledR}$ software, version 22.0 (SPSS, Inc, Chicago, IL). Shapiro-Wilk test, Kolmogorov-Smirnov test and normal probability plots were used to confirm the normal distribution of the data. Differences among the study groups were evaluated with the use of an analysis of variance (ANOVA) model, followed by the post hoc Bonferroni test when the findings with the ANOVA model were significant and equal variances assumed. When equal variances were not assumed, the significance was tested with Welch's $t$-test, followed by the post hoc GamesHowell test. For nonparametric variables, KruskalWallis test was conducted and significant values were adjusted with the Bonferroni correction, using Dunn posttest. A linear mixed model was designed to assess the status of Corvis ST parameters between different groups adjusting for confounding variables (age, gender, GATIOP, and prostaglandin analogues medication). The patient identification number was included as a random effect to correct for the inclusion of both eyes in some participants. Statistical significance was defined as $\mathrm{p}<0.05$.

\section{Results}

This study included 183 eyes of 115 patients: 61 with POAG, 32 with AG, 37 with OHT and 53 were healthy controls.

Demographic and baseline characteristics are shown in Table 1. Mean age was significantly lower in AG group compared with other groups (all $\mathrm{p}<0.001)$. Control group had lower mean age than POAG $(\mathrm{p}<0.001)$, and OHT $(p=0.001)$ groups. CCT was significantly higher in controls compared with POAG $(\mathrm{p}<0.001)$, and AG $(\mathrm{p}=0.016)$ groups. No differences were found in the number of glaucomatous medications after excluding healthy subjects.

\section{Primary Outcome}

The mean $\pm \mathrm{SD}$ and values of the mixed linear regression for biomechanical corneal parameters, adjusted for age, gender, GAT-IOP, and prostaglandin analogue medication, are shown in Tables 2-4.

Compared with controls (Table 2), AG eyes had smaller radius $(p=0.025)$, lower HCDeflA $(p=0.019)$, and higher $1 / R(p=0.014)$. There was a trend for a lower SSI $(p=0.09)$ in AG eyes, and a higher SPA1 $(p=0.07)$ in OHT eyes. OHT eyes had also higher SSI $(\mathrm{p}=0.12)$, and lower $1 /$ $\mathrm{R}(\mathrm{p}=0.33)$ than controls, but without reaching statistical significance. No differences were found between POAG eyes and healthy controls.

Compared with POAG eyes (Table 3), OHT eyes had higher SPA1 $(p=0.043)$, and $A G$ eyes had higher $1 / R$ $(p=0.010)$ and DA ratio $(p=0.025)$. There was a trend for a higher HCDeflA in AG eyes $(p=0.052)$ compared with POAG eyes.

Compared with AG eyes (Table 4), OHT had a lower HCDeflA $\quad(p=0.028), 1 / \mathrm{R} \quad(\mathrm{p}=0.004)$, and $\mathrm{DA}$ ratio $(p=0.01)$, and a higher SSI $(p=0.049)$.

These results are consistent with more deformable corneas in AG eyes, and less deformable corneas in OHT eyes.

\section{Secondary Outcome}

In this analysis, GAT-IOP, GAT-IOPadj, and bIOP were compared. CCT and bIOP were provided by Corvis ST.

As shown in Table 1, GAT-IOP was not significantly different between groups $(\mathrm{p}=0.171)$. The same was observed for GAT-IOPadj ( $\mathrm{p}=0.496)$, and bIOP $(\mathrm{p}=0.219)$. 
Table I Demographic and Baseline Characteristics of the Study Groups

\begin{tabular}{|c|c|c|c|c|c|}
\hline & Controls $(n=53)$ & POAG $(n=61)$ & AG $(n=32)$ & OHT $(n=37)$ & p-value \\
\hline Number of patients & 28 & 35 & 28 & 24 & - \\
\hline Age (y) & $62 \pm 10$ & $77 \pm 10$ & $53 \pm 8$ & $72 \pm 8$ & $p<0.001$ \\
\hline Male (\%) & $43 \%$ & $54 \%$ & $61 \%$ & $25 \%$ & $p=0.008$ \\
\hline Right eye (\%) & $51 \%$ & $51 \%$ & $41 \%$ & $46 \%$ & NS \\
\hline BCVA (logMAR) & $0.08 \pm 0.14$ & $0.15 \pm 0.22$ & $0.12 \pm 0.17$ & $0.08 \pm 0.12$ & $\mathrm{p}=0.036$ \\
\hline CCT $(\mu \mathrm{m})$ & $561 \pm 35$ & $533 \pm 34$ & $538 \pm 31$ & $547 \pm 28$ & $p<0.001$ \\
\hline GAT-IOP $(\mathrm{mmHg})$ & $14.02 \pm 2.18$ & $13.36 \pm 2.97$ & $12.91 \pm 2.90$ & $14.14 \pm 2.75$ & NS \\
\hline GAT-IOPadj (mmHg) & $13.57 \pm 3.45$ & $|4.43 \pm 3.5|$ & $13.66 \pm 3.44$ & $14.35 \pm 3.12$ & NS \\
\hline blOP (mmHg) & $13.22 \pm 2.38$ & $|3.00 \pm 4.7|$ & $13.68 \pm 3.53$ & $13.43 \pm 2.56$ & NS \\
\hline Number of glaucomatous medications & - & $2.15 \pm 0.93$ & $2.40 \pm 1.41$ & $1.84 \pm 0.80$ & NS \\
\hline Prostaglandin analogues & - & 44 (72\%) & $19(59 \%)$ & $22(59 \%)$ & NS \\
\hline$\beta$-blockers & - & 48 (79\%) & $25(78 \%)$ & 31 (84\%) & NS \\
\hline CA-inh & - & $30(49 \%)$ & $20(63 \%)$ & $10(27 \%)$ & $p=0.010$ \\
\hline$\alpha$ agonist & - & $12(20 \%)$ & 14 (44\%) & $5(14 \%)$ & $\mathrm{p}=0.008$ \\
\hline
\end{tabular}

Note: Values are presented as means \pm SD or $\mathrm{n}(\%)$.

Abbreviations: POAG, primary open-angle glaucoma; AG, amyloidotic glaucoma; OHT, ocular hypertension; $y$, years-old; BCVA, best-corrected visual acuity; logMAR, logarithm of the minimum angle of resolution; CCT, corneal central thickness; GAT-IOP, intraocular pressure measured by Goldmann applanation tonometry; GAT-IOPadj, intraocular pressure measured by Goldmann applanation tonometry and adjusted for pachymetry; bIOP, biomechanically corrected intraocular pressure; CA-inh, carbonic anhydrase inhibitors; $\mathrm{NS}$, non statically significant; $\mathrm{mmHg}$, millimeters of mercury; $\mu \mathrm{m}$, micrometers.

For each group, the differences between GAT-IOP, GAT-IOPadj, and bIOP are demonstrated in Figure 1. Biomechanically corrected IOP was significantly lower than GAT-IOP in POAG group $(13.00 \pm 4.71$ vs 13.36 $\pm 2.97 \mathrm{mmHg}, \mathrm{p}=0.005)$, and control group $(13.03 \pm 2.36$ vs $14.02 \pm 2.18 \mathrm{mmHg}, \mathrm{p}=0.013)$. The only group in which bIOP was higher than GAT-IOP was AG group (13.68 \pm 3.53 vs $12.91 \pm 2.90 \mathrm{mmHg}$ ), but without reaching statistical significance $(\mathrm{p}=0.46)$. In addition, bIOP was significantly lower than GAT-IOPadj in POAG group (13.00 \pm 4.71 vs $14.43 \pm 3.51 \mathrm{mmHg}, \mathrm{p}=0.01$ ).

\section{Discussion}

Corneal biomechanics has become an interesting topic in glaucoma. The glaucoma disease has a significant prevalence worldwide, ${ }^{16}$ and the search for a deeper understanding of glaucoma pathophysiology is ongoing. Our study was conducted to use recently developed technology in the evaluation of corneal biomechanical behavior in patients with POAG, OHT and AG. AG was included in this study because this type of secondary glaucoma is relatively prevalent in endemic regions for ATTR, like Portugal or Sweden, and the underlying mechanisms are very poorly understood.

Our findings were suggestive of more deformable corneas in AG eyes, and less deformable corneas in OHT eyes. Noteworthy, our results were obtained after adjusting for potential influencing factors of corneal biomechanics, like age, gender, GAT-IOP and prostaglandin analogue medication. ${ }^{17-20}$ Furthermore, there were significant differences between bIOP and GATIOP, which is the gold standard method for evaluating IOP, or GAT-IOPadj.

Many studies on corneal biomechanics in glaucoma have suggested that POAG corneas were less deformable than normal corneas. ${ }^{21-23}$ A lower corneal hysteresis $(\mathrm{CH})$ obtained with ORA device was found in POAG eyes which reflects a lower ability of the cornea to absorb and dissipate the mechanical energy applied by a deformation force. ${ }^{21}$ Using the Corvis ST, POAG eyes had lower deformation amplitude compared with healthy subjects. $^{22-24}$ Wang et $\mathrm{al}^{22}$ and Lee et $\mathrm{al}^{25}$ found 
Table 2 The Mean Values and Mixed Linear Model (Control Group Set as Reference) for Corvis ST Parameters Adjusted for Age, Gender, GAT-IOP, and Prostaglandin Analogue Medication

\begin{tabular}{|c|c|c|c|c|}
\hline & Controls $(n=53)$ & POAG $(n=6 I)$ & AG $(n=32)$ & OHT $(n=37)$ \\
\hline \multicolumn{5}{|c|}{$\mathrm{AIT}(\mathrm{ms})$} \\
\hline Mean $\pm S D$ & $7.7 I \pm 0.29$ & $7.68 \pm 0.67$ & $7.67 \pm 0.49$ & $7.78 \pm 0.37$ \\
\hline Coefficient \pm SE & \multirow[t]{2}{*}{ Reference } & $0.073 \pm 0.164$ & $-0.078 \pm 0.153$ & $0.142 \pm 0.162$ \\
\hline $\mathrm{p}$-value & & NS & NS & NS \\
\hline \multicolumn{5}{|c|}{$\operatorname{AIV}(\mathrm{m} / \mathrm{s})$} \\
\hline Mean $\pm S D$ & $0.138 \pm 0.019$ & $0.142 \pm 0.021$ & $0.142 \pm 0.022$ & $0.137 \pm 0.024$ \\
\hline Coefficient $\pm S E$ & \multirow[t]{2}{*}{ Reference } & $-0.001 \pm 0.007$ & $0.00005 \pm 0.006$ & $-0.007 \pm 0.007$ \\
\hline $\mathrm{p}$-value & & NS & NS & NS \\
\hline \multicolumn{5}{|c|}{ AIDeflL (mm) } \\
\hline Mean $\pm S D$ & $2.29 \pm 0.22$ & $2.28 \pm 0.16$ & $2.21 \pm 0.18$ & $2.28 \pm 0.14$ \\
\hline Coefficient \pm SE & \multirow[t]{2}{*}{ Reference } & $-0.01 I \pm 0.053$ & $-0.049 \pm 0.050$ & $-0.010 \pm .052$ \\
\hline $\mathrm{p}$-value & & NS & NS & NS \\
\hline \multicolumn{5}{|c|}{ PD (mm) } \\
\hline Mean $\pm S D$ & $4.77 \pm 0.31$ & $4.87 \pm 0.48$ & $4.85 \pm 0.47$ & $4.74 \pm 0.32$ \\
\hline Coefficient $\pm S E$ & \multirow[t]{2}{*}{ Reference } & $0.112 \pm 0.138$ & $0.161 \pm 0.128$ & $-0.036 \pm 0.136$ \\
\hline $\mathrm{p}$-value & & NS & NS & NS \\
\hline \multicolumn{5}{|c|}{ Radius (mm) } \\
\hline Mean $\pm S D$ & $6.83 \pm 1.23$ & $6.56 \pm 0.91$ & $6.14 \pm 0.62$ & $6.44 \pm 0.74$ \\
\hline Coefficient $\pm S E$ & \multirow[t]{2}{*}{ Reference } & $-0.081 \pm 0.260$ & $-0.552 \pm 0.247$ & $-0.171 \pm 0.253$ \\
\hline $\mathrm{p}$-value & & NS & 0.025 & NS \\
\hline \multicolumn{5}{|c|}{ HCDA (mm) } \\
\hline Mean $\pm S D$ & $1.05 \pm 0.11$ & $1.05 \pm 0.16$ & $1.04 \pm 0.21$ & $1.02 \pm 0.10$ \\
\hline Coefficient $\pm S E$ & \multirow[t]{2}{*}{ Reference } & $-0.028 \pm 0.050$ & $0.034 \pm 0.046$ & $-0.047 \pm 0.049$ \\
\hline $\mathrm{p}$-value & & NS & NS & NS \\
\hline \multicolumn{5}{|c|}{ HCDefIA (mm) } \\
\hline Mean $\pm S D$ & $0.756 \pm 0.601$ & $0.887 \pm 0.15$ & $0.879 \pm 0.192$ & $0.840 \pm 0.107$ \\
\hline Coefficient \pm SE & \multirow[t]{2}{*}{ Reference } & $0.011 \pm 0.096$ & $0.213 \pm 0.091$ & $-0.012 \pm 0.093$ \\
\hline $\mathrm{p}$-value & & NS & 0.019 & NS \\
\hline \multicolumn{5}{|c|}{$\mathrm{A} 2 \mathrm{~T}(\mathrm{~ms})$} \\
\hline Mean \pm SD & $21.94 \pm 0.46$ & $21.87 \pm 0.66$ & $22.11 \pm 0.67$ & $21.86 \pm 0.49$ \\
\hline Coefficient \pm SE & \multirow[t]{2}{*}{ Reference } & $-0.063 \pm 0.196$ & $0.248 \pm 0.181$ & $-0.086 \pm 0.192$ \\
\hline $\mathrm{p}$-value & & NS & NS & NS \\
\hline
\end{tabular}

(Continued) 
Table 2 (Continued).

\begin{tabular}{|c|c|c|c|c|}
\hline & Controls $(n=53)$ & POAG $(n=6 I)$ & AG $(n=32)$ & OHT $(n=37)$ \\
\hline \multicolumn{5}{|c|}{$\mathrm{A} 2 \mathrm{~V}(\mathrm{~m} / \mathrm{s})$} \\
\hline Mean $\pm S D$ & $-0.23 \pm 0.04$ & $-0.26 \pm 0.05$ & $-0.25 \pm 0.07$ & $-0.25 \pm 0.03$ \\
\hline Coefficient \pm SE & \multirow[t]{2}{*}{ Reference } & $-0.013 \pm 0.015$ & $-0.010 \pm 0.014$ & $0.001 \pm 0.015$ \\
\hline $\mathrm{p}$-value & & NS & NS & NS \\
\hline \multicolumn{5}{|c|}{ A2DeflL (mm) } \\
\hline Mean \pm SD & $3.3| \pm 0.7|$ & $3.01 \pm 0.74$ & $2.91 \pm 0.85$ & $2.96 \pm 0.59$ \\
\hline Coefficient \pm SE & \multirow[t]{2}{*}{ Reference } & $-0.225 \pm 0.196$ & $-0.188 \pm 0.188$ & $-0.206 \pm 0.191$ \\
\hline $\mathrm{p}$-value & & NS & NS & NS \\
\hline \multicolumn{5}{|c|}{ SSI } \\
\hline Mean \pm SD & $1.244 \pm 0.174$ & $1.308 \pm 0.232$ & $1.119 \pm 0.324$ & $1.322 \pm 0.187$ \\
\hline Coefficient \pm SE & \multirow[t]{2}{*}{ Reference } & $-0.0|4 \pm 0.08|$ & $-0.127 \pm 0.075$ & $0.03 I \pm 0.079$ \\
\hline $\mathrm{p}$-value & & NS & 0.090 & NS \\
\hline \multicolumn{5}{|c|}{ SPAI } \\
\hline Mean $\pm S D$ & $110.30 \pm 22.98$ & $109.03 \pm 19.24$ & $108.23 \pm 25.90$ & $119.90 \pm 19.84$ \\
\hline Coefficient \pm SE & \multirow[t]{2}{*}{ Reference } & $1.830 \pm 7.028$ & $5.687 \pm 6.494$ & $12.487 \pm 6.890$ \\
\hline $\mathrm{P}$-value & & NS & NS & 0.070 \\
\hline \multicolumn{5}{|c|}{$\mathrm{I} / \mathrm{R}$} \\
\hline Mean \pm SD & $8.69 \pm 0.78$ & $9.13 \pm 1.51$ & $9.66 \pm 1.57$ & $8.96 \pm 1.14$ \\
\hline Coefficient \pm SE & \multirow[t]{2}{*}{ Reference } & $-0.137 \pm 0.431$ & $0.983 \pm 0.399$ & $-0.249 \pm 0.424$ \\
\hline $\mathrm{p}$-value & & NS & 0.014 & NS \\
\hline \multicolumn{5}{|c|}{ DARatio } \\
\hline Mean \pm SD & $1.62 \pm 0.57$ & $1.56 \pm 0.05$ & $1.55 \pm 0.09$ & $1.55 \pm 0.06$ \\
\hline Coefficient $\pm S E$ & \multirow[t]{2}{*}{ Reference } & $-0.125 \pm 0.152$ & $0.225 \pm 0.141$ & $-0.169 \pm 0.149$ \\
\hline$p$-value & & NS & NS & NS \\
\hline
\end{tabular}

Abbreviations: POAG, primary open-angle glaucoma; AG, amyloidotic glaucoma; OHT, ocular hypertension; SD, standard deviation; SE, standard error; NS, no statistical difference; AIT, time at first applanation; AIV, velocity at first applanation; AIDefiL, Deflection length at first applanation; PD, peak distance; HCDA, deformation amplitude at highest concavity; HCDeflA, deflection amplitude at highest concavity; A2T, time at second applanation; A2V, velocity at second applanation; A2DeflL, deflection length at second applanation; SSI, stress-strain index; SPAI, stiffness parameter at first applanation; I/R, integrated radius; DARatio, deformation amplitude ration; mm, millimeters; ms, milliseconds; GAT-IOP, Intraocular pressure measured by Goldmann applanation tonometry; PG, prostaglandin analogues.

a greater $\mathrm{A} 2 \mathrm{~V}$ and $\mathrm{PD}$ (indicating a stiffer cornea) in glaucomatous eyes. In contrast, Miki et $\mathrm{al}^{26}$ reported a smaller A1T and Rad consistent with more deformable corneas in medically controlled glaucomatous eyes and the lack of control for confounding factors in previous studies was pointed out as the cause for the discrepant results. In agreement with our study, Pradhan et $\mathrm{al}^{27}$ demonstrated a similar corneal biomechanical profile between POAG patients and healthy subjects. Eyes under anti-glaucoma medication were excluded from this study. In fact, there is evidence that prostaglandin analogues affect the corneal biomechanical properties by activation of matrix metalloproteinases, resulting in a lower $\mathrm{CH}$ and higher deformation amplitude. ${ }^{19,20}$ In our work, as in that by Pradhan 
Table 3 Mixed Linear Model (Primary Open-Angle Glaucoma Group Set as Reference) for Corvis ST Parameters Adjusted for Age, Gender, GAT-IOP, and Prostaglandin Analogue Medication

\begin{tabular}{|c|c|c|c|c|}
\hline & Controls $(n=53)$ & POAG $(n=6 I)$ & AG $(n=32)$ & OHT $(n=37)$ \\
\hline \multicolumn{5}{|c|}{ AIT (ms) } \\
\hline Coefficient $\pm S E$ & $-0.072 \pm 0.164$ & \multirow[t]{2}{*}{ Reference } & $-0.15 I \pm 0.164$ & $0.0696 \pm 0.113$ \\
\hline $\mathrm{p}$-value & NS & & NS & NS \\
\hline \multicolumn{5}{|c|}{$\operatorname{AIV}(\mathrm{m} / \mathrm{s})$} \\
\hline Coefficient $\pm S E$ & $0.00 I \pm 0.007$ & \multirow[t]{2}{*}{ Reference } & $0.00 I \pm 0.007$ & $-0.006 \pm 0.005$ \\
\hline $\mathrm{p}$-value & NS & & NS & NS \\
\hline \multicolumn{5}{|c|}{ AIDeflL (mm) } \\
\hline Coefficient \pm SE & $0.0115 \pm 0.054$ & \multirow[t]{2}{*}{ Reference } & $-0.037 \pm 0.057$ & $-0.037 \pm 0.057$ \\
\hline $\mathrm{p}$-value & NS & & NS & NS \\
\hline \multicolumn{5}{|c|}{$\mathrm{PD}(\mathrm{mm})$} \\
\hline Coefficient \pm SE & $-0.112 \pm 0.138$ & \multirow[t]{2}{*}{ Reference } & $0.049 \pm 0.138$ & $-0.148 \pm 0.097$ \\
\hline $\mathrm{p}$-value & NS & & NS & NS \\
\hline \multicolumn{5}{|c|}{ Radius (mm) } \\
\hline Coefficient \pm SE & $0.08 I \pm 0.259$ & \multirow[t]{2}{*}{ Reference } & $-0.47 I \pm 0.282$ & $-0.090 \pm 0.205$ \\
\hline$p$-value & NS & & $*_{p}=0.095$ & NS \\
\hline \multicolumn{5}{|c|}{$\mathrm{HCDA}(\mathrm{mm})$} \\
\hline Coefficient \pm SE & $0.028 \pm 0.050$ & \multirow[t]{2}{*}{ Reference } & $0.062 \pm 0.050$ & $-0.019 \pm 0.035$ \\
\hline $\mathrm{p}$-value & NS & & NS & NS \\
\hline \multicolumn{5}{|c|}{ HCDeflA (mm) } \\
\hline Coefficient \pm SE & $-0.011 \pm 0.096$ & \multirow[t]{2}{*}{ Reference } & $0.202 \pm 0.104$ & $-0.023 \pm 0.076$ \\
\hline $\mathrm{p}$-value & NS & & $*_{p}=0.052$ & NS \\
\hline \multicolumn{5}{|c|}{ A2T (ms) } \\
\hline Coefficient \pm SE & $0.063 \pm 0.196$ & \multirow[t]{2}{*}{ Reference } & $0.311 \pm 0.198$ & $-0.023 \pm 0.142$ \\
\hline $\mathrm{p}$-value & NS & & NS & NS \\
\hline \multicolumn{5}{|c|}{$\mathrm{A} 2 \mathrm{~V}(\mathrm{~m} / \mathrm{s})$} \\
\hline Coefficient \pm SE & $0.013 \pm 0.015$ & \multirow[t]{2}{*}{ Reference } & $0.003 \pm 0.016$ & $0.014 \pm 0.012$ \\
\hline $\mathrm{p}$-value & NS & & NS & NS \\
\hline \multicolumn{5}{|c|}{ A2DeflL (mm) } \\
\hline Coefficient \pm SE & $0.225 \pm 0.196$ & \multirow[t]{2}{*}{ Reference } & $0.036 \pm 0.215$ & $0.019 \pm 0.155$ \\
\hline $\mathrm{p}$-value & NS & & NS & NS \\
\hline \multicolumn{5}{|c|}{ SSI } \\
\hline Coefficient \pm SE & $0.014 \pm 0.081$ & \multirow[t]{2}{*}{ Reference } & $-0.113 \pm 0.082$ & $0.045 \pm 0.058$ \\
\hline $\mathrm{p}$-value & NS & & NS & NS \\
\hline
\end{tabular}


Table 3 (Continued).

\begin{tabular}{|c|c|c|c|c|}
\hline & Controls $(n=53)$ & POAG $(n=61)$ & AG $(n=32)$ & OHT $(n=37)$ \\
\hline \multicolumn{5}{|c|}{ SPAI } \\
\hline Coefficient $\pm S E$ & $-1.83 \pm 7.02$ & \multirow[t]{2}{*}{ Reference } & $3.857 \pm 7.173$ & $10.657 \pm 5.277$ \\
\hline $\mathrm{p}$-value & NS & & NS & $p=0.043$ \\
\hline \multicolumn{5}{|c|}{$\mathrm{I} / \mathrm{R}$} \\
\hline Coefficient \pm SE & $0.136 \pm 0.431$ & \multirow[t]{2}{*}{ Reference } & $1.120 \pm 0.434$ & $-0.112 \pm 0.310$ \\
\hline $\mathrm{p}$-value & NS & & $p=0.010$ & NS \\
\hline \multicolumn{5}{|c|}{ DARatio } \\
\hline Coefficient \pm SE & $0.125 \pm 0.152$ & \multirow[t]{2}{*}{ Reference } & $0.350 \pm 0.156$ & $-0.044 \pm 0.115$ \\
\hline $\mathrm{p}$-value & NS & & $p=0.025$ & NS \\
\hline
\end{tabular}

Note: *NS, but a tendency to statistical significance.

Abbreviations: POAG, primary open-angle glaucoma; AG, amyloidotic glaucoma; OHT, ocular hypertension; SD, standard deviation; SE, standard error; NS, no statistical difference; AIT, time at first applanation; AIV, velocity at first applanation; AIDeflL, deflection length at first applanation; PD, peak distance; HCDA, deformation amplitude at highest concavity; HCDeflA, deflection amplitude at highest concavity; A2T, time at second applanation; A2V, velocity at second applanation; A2DeflL, deflection length at second applanation; SSI, stress strain index; SPAI, stiffness parameter at first applanation; I/R, integrated radius; DARatio, deformation amplitude ration; mm, millimeters; ms, milliseconds; GAT-IOP, Intraocular pressure measured by Goldmann applanation tonometry; PG, prostaglandin analogues.

et $\mathrm{al}^{27}$ the effect of prostaglandin analogues in corneal biomechanics was taken into consideration, which may explain the similar results. Additionally, the present study is one of the few that analyzed the novel mathematicallyderived parameters (DA ratio, 1/R, SPA1, and SSI) in corneas of glaucoma eyes. ${ }^{12,28}$ It was proposed that these parameters were less dependent of IOP, ${ }^{29,30}$ and correlated with corneal biomechanics (including $\mathrm{CH}$ ). ${ }^{28}$ When these novel parameters were assessed, POAG group did not display a different corneal biomechanical behavior compared with controls, which is in line with previous published research. ${ }^{12,28}$

OHT has also been studied with this recent technology. A higher $\mathrm{CH}$ was documented in patients with OHT as compared to those with glaucoma. ${ }^{31,32}$ A recent study demonstrated a higher A1DeflL and A2V, and lower A1V compatible with stiffer corneas in OHT eyes, while we found a higher SSI. ${ }^{33}$ The differences in corneal biomechanics between OHT eyes and those with glaucoma, either POAG or AG, were evident in the present study. Moreover, OHT eyes usually present a thicker CCT. ${ }^{32}$ Thus, eyes with OHT can be less susceptible to ONH damage due to biomechanical properties of ocular structures, including peripapillary sclera. A thicker and stiffer peripapillary sclera can be a protective feature of ONH damage in OHT eyes.

AG is a secondary open-angle glaucoma that develops in patients with ATTR. Although glaucoma affects about
$20 \%$ of ATTR patients, ${ }^{34,35}$ this disease has been little explored in the research. For unknown reasons, the course of $\mathrm{AG}$ is usually accelerated and often requires surgical treatment. $^{36,37}$ Importantly, we found thinner and more deformable corneas in AG eyes which may represent an increased susceptibility to $\mathrm{ONH}$ damage. Hence, the rapid progression of AG may be related to the presence of a thinner and more deformable peripapillary sclera. Recent data suggested that more compliant corneas have a greater risk of glaucoma progression. ${ }^{38}$ In addition, bIOP was on average higher than GAT-IOP only in AG eyes, which may have important clinical implications. We hypothesize that, in this subset of patients, it may be reasonable to measure bIOP when there is rapid progression of glaucoma despite apparently controlled IOP. It is possible that biomechanical properties of amyloidotic eyes are at least partially responsible for glaucoma onset and aggressive progression.

To summarize, this study did not corroborate previous research which demonstrated less deformable corneas in POAG eyes compared with controls. However, OHT corneas seem to be less deformable than POAG corneas, as shown by a higher SPA1. In contrast, AG corneas seem to be more deformable than POAG corneas, as demonstrated by a higher $1 / \mathrm{R}$ and $\mathrm{DA}$ ratio.

In agreement with a study by Vinciguerra et al, ${ }^{12}$ bIOP was significantly lower than GAT-IOP and GAT-IOPadj in 
Table 4 Mixed Linear Model (Amyloidotic Glaucoma Group Set as Reference) for Corvis ST Parameters Adjusted for Age, Gender, GAT-IOP, and Prostaglandin Analogue Medication

\begin{tabular}{|c|c|c|c|c|}
\hline & $\begin{array}{l}\text { Controls } \\
(n=53)\end{array}$ & $\begin{array}{l}\text { POAG } \\
(n=61)\end{array}$ & $\begin{array}{c}A G \\
(n=32)\end{array}$ & $\begin{array}{c}\text { OHT } \\
(n=37)\end{array}$ \\
\hline \multicolumn{5}{|c|}{ AIT (ms) } \\
\hline Coefficient \pm SE & $0.078 \pm 0.153$ & $0.15 I \pm 0.164$ & \multirow[t]{2}{*}{ Reference } & $0.220 \pm 0.162$ \\
\hline $\mathrm{p}$-value & NS & NS & & NS \\
\hline \multicolumn{5}{|c|}{$\operatorname{AIV}(\mathrm{m} / \mathrm{s})$} \\
\hline Coefficient \pm SE & $-0.000 I \pm 0.006$ & $-0.00 I \pm 0.007$ & \multirow[t]{2}{*}{ Reference } & $-0.007 \pm 0.007$ \\
\hline $\mathrm{P}$-value & NS & NS & & NS \\
\hline \multicolumn{5}{|c|}{ AIDeflL (mm) } \\
\hline Coefficient $\pm S E$ & $0.049 \pm 0.050$ & $0.037 \pm 0.057$ & \multirow[t]{2}{*}{ Reference } & $0.038 \pm 0.056$ \\
\hline $\mathrm{p}$-value & NS & NS & & NS \\
\hline \multicolumn{5}{|c|}{$\mathrm{PD}(\mathrm{mm})$} \\
\hline Coefficient \pm SE & $-0.161 \pm 0.128$ & $-0.049 \pm 0.138$ & \multirow[t]{2}{*}{ Reference } & $-0.197 \pm 0.137$ \\
\hline $\mathrm{p}$-value & NS & NS & & NS \\
\hline \multicolumn{5}{|c|}{ Radius (mm) } \\
\hline Coefficient \pm SE & $0.55 I \pm 0.247$ & $0.47 I \pm 0.282$ & \multirow[t]{2}{*}{ Reference } & $0.38 I \pm 0.277$ \\
\hline $\mathrm{p}$-value & $p=0.025$ & $*_{p}=0.095$ & & NS \\
\hline \multicolumn{5}{|c|}{$\mathrm{HCDA}(\mathrm{mm})$} \\
\hline Coefficient \pm SE & $-0.034 \pm 0.046$ & $-0.062 \pm 0.050$ & \multirow[t]{2}{*}{ Reference } & $-0.081 \pm 0.049$ \\
\hline $\mathrm{p}$-value & NS & NS & & $*_{p}=0.098$ \\
\hline \multicolumn{5}{|c|}{ HCDeflA (mm) } \\
\hline Coefficient \pm SE & $-0.213 \pm 0.091$ & $-0.202 \pm 0.104$ & \multirow[t]{2}{*}{ Reference } & $-0.225 \pm 0.102$ \\
\hline $\mathrm{p}$-value & $p=0.019$ & $*_{p}=0.052$ & & $p=0.028$ \\
\hline \multicolumn{5}{|c|}{$\mathrm{A} 2 \mathrm{~T}(\mathrm{~ms})$} \\
\hline Coefficient \pm SE & $-0.248 \pm 0.181$ & $-0.31 I \pm 0.198$ & \multirow[t]{2}{*}{ Reference } & $-0.334 \pm 0.195$ \\
\hline $\mathrm{p}$-value & NS & NS & & $*_{p}=0.087$ \\
\hline \multicolumn{5}{|c|}{$\mathrm{A} 2 \mathrm{~V}(\mathrm{~m} / \mathrm{s})$} \\
\hline Coefficient \pm SE & $0.010 \pm 0.014$ & $-0.003 \pm 0.016$ & \multirow[t]{2}{*}{ Reference } & $0.011 \pm 0.015$ \\
\hline $\mathrm{p}$-value & NS & NS & & NS \\
\hline \multicolumn{5}{|c|}{ A2DeflL (mm) } \\
\hline Coefficient \pm SE & $0.188 \pm 0.188$ & $-0.036 \pm 0.215$ & \multirow[t]{2}{*}{ Reference } & $-0.017 \pm 0.211$ \\
\hline $\mathrm{p}$-value & NS & NS & & NS \\
\hline
\end{tabular}


Table 4 (Continued).

\begin{tabular}{|c|c|c|c|c|}
\hline & $\begin{array}{c}\text { Controls } \\
(n=53)\end{array}$ & $\begin{array}{l}\text { POAG } \\
(n=61)\end{array}$ & $\begin{array}{c}\text { AG } \\
(n=32)\end{array}$ & $\begin{array}{c}\text { OHT } \\
(n=37)\end{array}$ \\
\hline \multicolumn{5}{|c|}{ SSI } \\
\hline Coefficient \pm SE & $0.127 \pm 0.075$ & $0.113 \pm 0.082$ & \multirow[t]{2}{*}{ Reference } & $0.158 \pm 0.080$ \\
\hline $\mathrm{p}$-value & $*_{p}=0.090$ & NS & & $p=0.049$ \\
\hline \multicolumn{5}{|c|}{ SPAI } \\
\hline Coefficient \pm SE & $-5.687 \pm 6.493$ & $-3.857 \pm 7.173$ & \multirow[t]{2}{*}{ Reference } & $6.800 \pm 7.066$ \\
\hline $\mathrm{p}$-value & NS & NS & & NS \\
\hline \multicolumn{5}{|c|}{$\mathrm{I} / \mathrm{R}$} \\
\hline Coefficient $\pm S E$ & $-0.983 \pm 0399$ & $-1.120 \pm 0.434$ & \multirow[t]{2}{*}{ Reference } & $-1.232 \pm 0.428$ \\
\hline $\mathrm{p}$-value & $\mathrm{p}=0.014$ & $\mathrm{P}=0.010$ & & $\mathrm{p}=0.004$ \\
\hline \multicolumn{5}{|c|}{ DARatio } \\
\hline Coefficient \pm SE & $-0.225 \pm 0.141$ & $-0.350 \pm 0.156$ & \multirow[t]{2}{*}{ Reference } & $-0.394 \pm 0.153$ \\
\hline$P$-value & NS & $\mathrm{P}=0.025$ & & $P=0.01$ \\
\hline
\end{tabular}

Note: *NS, but a tendency to statistical significance.

Abbreviations: POAG, primary open-angle glaucoma; AG, amyloidotic glaucoma; OHT, ocular hypertension; SD, standard deviation; SE, standard error; NS, no statistical difference; AIT, time at first applanation; AIV, velocity at first applanation; AIDeflL, Deflection length at first applanation; PD, peak distance; HCDA, deformation amplitude at highest concavity; HCDeflA, deflection amplitude at highest concavity; A2T, time at second applanation; A2V, velocity at second applanation; A2DeflL, deflection length at second applanation; SSI, stress strain index; SPAI, stiffness parameter at first applanation; I/R, integrated radius; DARatio, deformation amplitude ration; mm, millimeters; ms, milliseconds; GAT-IOP, Intraocular pressure measured by Goldmann applanation tonometry; PG, prostaglandin analogues.

POAG patients. Likewise, GAT-IOP adjusted for CCT and bIOP do not seem to be interchangeable. Corneal compensated IOP (IOPcc) is a biomechanical parameter assessed by the ORA device, comparable to bIOP provided by the Corvis ST. Two studies ${ }^{39,40}$ reported a higher value of IOPcc compared with GAT-IOP. This led us to note the disparity between the comparison of GAT-IOP with these two types of biomechanically corrected IOP, IOPcc (ORA device) and bIOP (Corvis ST device). Matsuura et $\mathrm{al}^{41}$

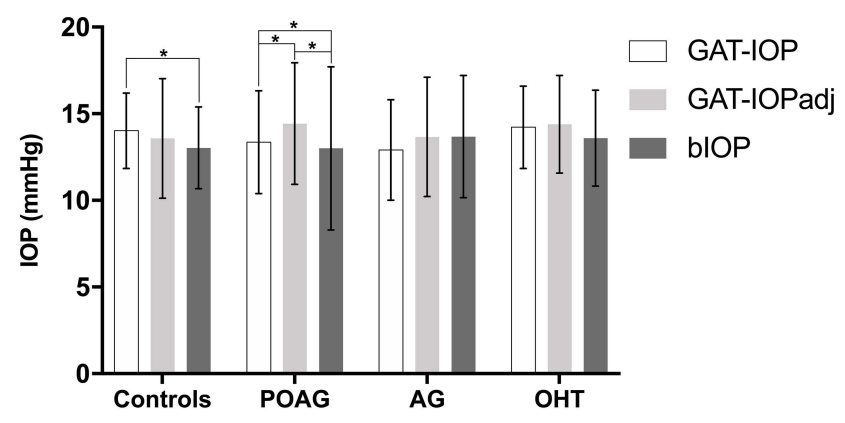

Figure I The mean values of intraocular pressure (IOP) obtained by Goldmann applanation tonometry (GAT-IOP), GAT-IOP corrected for pachymetry (GATIOPadj), and biomechanically corrected IOP (bIOP) in study groups: control group, primary open-angle glaucoma (POAG) group, amyloidotic glaucoma (AG) group, and ocular hypertension (OHT) group. ${ }^{*} \mathrm{p}<0.05$. corroborated these findings showing that bIOP is lower than IOPcc and GAT-IOP. In intracameral studies, ${ }^{42-44}$ true IOP was usually higher than GAT-IOP, such as IOPcc, but not bIOP. Thus, there may not be a relationship between bIOP and true IOP. However, an ex vivo study showed no differences between bIOP and internal manometric IOP $(0.3 \pm 1.6 \mathrm{mmHg}, \mathrm{p}=0.989)$ in 5 ocular globes of humans. ${ }^{45}$

This study has limitations. First, the cross-sectional nature of our study design prevented the evaluation of causality or prospective prediction of glaucoma risk. All glaucoma patients were under topical treatment, and, to minimize the effect of prostaglandin analogues in corneal biomechanics, it was regarded as a confounding factor in mixed linear regression. Of note, we performed a statistical correction for the inclusion of both eyes in some study participants. Some subjects were previously submitted to ophthalmic surgery, but a minimal interval of 6 months was imposed by inclusion criteria to overcome this limitation. The influence on the results is expected to be minimal after this period. ${ }^{46,47}$ Afterwards, it was not possible to assess the glaucoma severity due to unavailability of automated perimetry at the time of the study. Finally, it should be noted that the sclera and cornea differ 
on the dimensions and arrangement of collagen fibrils, which may lead to distinct biomechanical behavior. ${ }^{4}$ This underlines the importance of in vivo methods for assessing scleral and LC structural properties to become available.

As strengths of this study, we underline the interesting and novel findings in the group of AG eyes. To our knowledge, no other study has analyzed simultaneously so many corneal biomechanical parameters. This comparative study exposed the differences in biomechanical behavior of eyes with POAG, OHT and AG, and supports the role of ocular biomechanics in individual susceptibility to glaucomatous damage. Therapeutic approaches aiming to modify biomechanical properties of peripapillary sclera have emerged, and this kind of studies will help to establish a rationale for them. ${ }^{48}$ Moreover, different IOP measurements provided different values, and it became evident that further investigation is needed to support the use of IOP corrected for corneal biomechanics.

To conclude, our results were suggestive of more deformable corneas in AG eyes, and less deformable corneas in OHT. Thus, AG eyes seem to be more susceptible to glaucomatous damage and progression, while OHT eyes seem to be more resistant to $\mathrm{ONH}$ damage. There were significant differences between GAT-IOP, GAT-IOP adjusted for CCT and bIOP. Further investigation is needed to validate the use of bIOP.

\section{Ethics}

The study was conducted according to the tenets of the Declaration of Helsinki in its latest amendment (Brazil, 2013) and was approved by the local IRB ("Departamento de Ensino, Formação e Investigação" of Centro Hospitalar Universitário do Porto).

\section{Informed Consent}

All patients signed an informed consent form.

\section{Author Contributions}

All authors made a significant contribution to the work reported, whether that is in the conception, study design, execution, acquisition of data, analysis and interpretation, or in all these areas; took part in drafting, revising or critically reviewing the article; gave final approval of the version to be published; have agreed on the journal to which the article has been submitted; and agree to be accountable for all aspects of the work.

\section{Funding}

The authors have no funding to declare.

\section{Disclosure}

Dr Riccardo Vinciguerra reports personal fees from OCULUS, during the conduct of the study. The authors have no other conflicts of interest to declare.

\section{References}

1. Brown KE, Congdon NG. Corneal structure and biomechanics: impact on the diagnosis and management of glaucoma. Curr Opin Ophthalmol. 2006;17(4):338-343. doi:10.1097/01.icu.0000233951.01971.5b

2. Coleman AL, Miglior S. Risk factors for glaucoma onset and progression. Surv Ophthalmol. 2008;53(Suppl1):S3-10. doi:10.10 16/j.survophthal.2008.08.006

3. Burgoyne CF, Downs JC, Bellezza AJ, Suh JK, Hart RT. The optic nerve head as a biomechanical structure: a new paradigm for understanding the role of IOP-related stress and strain in the pathophysiology of glaucomatous optic nerve head damage. Prog Retin Eye Res. 2005;24(1):39-73. doi:10.1016/j.preteyeres.2004.06.001

4. Komai Y, Ushiki T. The three-dimensional organization of collagen fibrils in the human cornea and sclera. Invest Ophthalmol Vis Sci. 1991;32(8):2244-2258.

5. Congdon NG, Broman AT, Bandeen-Roche K, Grover D, Quigley HA. Central corneal thickness and corneal hysteresis associated with glaucoma damage. Am J Ophthalmol. 2006;141 (5):868-875. doi:10.1016/j.ajo.2005.12.007

6. Medeiros FA, Meira-Freitas D, Lisboa R, Kuang TM, Zangwill LM, Weinreb RN. Corneal hysteresis as a risk factor for glaucoma progression: a prospective longitudinal study. Ophthalmology. 2013;120 (8):1533-1540. doi:10.1016/j.ophtha.2013.01.032

7. Yanoff M, Duker JS, Augsburger JJ. Ophthalmology. 2nd ed. St Louis: Missouri: Mosby; 2004.

8. European Glaucoma Society Terminology and Guidelines for Glaucoma, 4th Edition - Part 1 Supported by the EGS Foundation. Br J Ophthalmol. 2017;101(4):1-72. doi:10.1136/bjophthalmol-2016EGSguideline.001

9. Ehlers N, Bramsen T, Sperling S. Applanation tonometry and central corneal thickness. Acta Ophthalmol (Copenh). 1975;53(1):34-43. doi:10.1111/j.1755-3768.1975.tb01135.x

10. Holladay JT. Proper method for calculating average visual acuity. J Refract Surg. 1997;13(4):388-391. doi:10.3928/1081-597X-19970701-16

11. Salvetat ML, Zeppieri M, Tosoni C, Felletti M, Grasso L, Brusini P. Corneal deformation parameters provided by the Corvis-ST Pachy-Tonometer in healthy subjects and glaucoma patients. J Glaucoma. 2015;24(8):568-574. doi:10.1097/IJG.0000000000000133

12. Vinciguerra R, Rehman S, Vallabh NA, et al. Corneal biomechanics and biomechanically corrected intraocular pressure in primary open-angle glaucoma, ocular hypertension and controls. Br J Ophthalmol. 2020;104 (1):121-126. doi:10.1136/bjophthalmol-2018-313493

13. Eliasy A, Chen KJ, Vinciguerra R, et al. Determination of corneal biomechanical behavior in-vivo for healthy eyes using CorVis ST tonometry: stress-strain index. Front Bioeng Biotechnol. 2019;7:105. doi:10.3389/fbioe.2019.00105

14. Nemeth G, Hassan Z, Csutak A, Szalai E, Berta A, Modis L Jr. Repeatability of ocular biomechanical data measurements with a Scheimpflug-based noncontact device on normal corneas. J Refract Surg. 2013;29(8):558-563. doi:10.3928/1081597X-20130719-06

15. Lopes BT, Roberts CJ, Elsheikh A, et al. Repeatability and reproducibility of intraocular pressure and dynamic corneal response parameters assessed by the Corvis ST. J Ophthalmol. 2017;20 17:8515742. doi: $10.1155 / 2017 / 8515742$ 
16. Tham YC, Li X, Wong TY, Quigley HA, Aung T, Cheng CY. Global prevalence of glaucoma and projections of glaucoma burden through 2040: a systematic review and meta-analysis. Ophthalmology. 2014;121(11):2081-2090. doi:10.1016/j.ophtha.2014.05.013

17. Huseynova T, Waring G, Roberts C, Krueger RR, Tomita M. Corneal biomechanics as a function of intraocular pressure and pachymetry by dynamic infrared signal and Scheimpflug imaging analysis in normal eyes. Am J Ophthalmol. 2014;157(4):885-893. doi:10.1016/ j.ajo.2013.12.024

18. Roberts CJ. Concepts and misconceptions in corneal biomechanics. $J$ Cataract Refract Surg. 2014;40(6):862-869. doi:10.1016/j. jcrs.2014.04.019

19. Wu N, Chen Y, Yu X, Li M, Wen W, Sun X. Changes in corneal biomechanical properties after long-term topical prostaglandin therapy. PLoS One. 2016;11(5):e0155527. doi:10.1371/journal. pone. 0155527

20. Meda R, Wang Q, Paoloni D, Harasymowycz P, Brunette I. The impact of chronic use of prostaglandin analogues on the biomechanical properties of the cornea in patients with primary open-angle glaucoma. Br J Ophthalmol. 2017;101(2):120-125. doi:10.1136/ bjophthalmol-2016-308432

21. Ang GS, Bochmann F, Townend J, Azuara-Blanco A. Corneal biomechanical properties in primary open angle glaucoma and normal tension glaucoma. J Glaucoma. 2008;17(4):259-262. doi:10.1097/ IJG.0b013e31815c3a93

22. Wang W, Du S, Zhang X. Corneal deformation response in patients with primary open-angle glaucoma and in healthy subjects analyzed by Corvis ST. Invest Ophthalmol Vis Sci. 2015;56(9):5557-5565. doi:10.1167/iovs.15-16926

23. Tian L, Wang D, Wu Y, et al. Corneal biomechanical characteristics measured by the CorVis Scheimpflug technology in eyes with primary open-angle glaucoma and normal eyes. Acta Ophthalmol. 2016;94(5):e317-24. doi:10.1111/aos.12672

24. Jung Y, Park HL, Yang HJ, Park CK. Characteristics of corneal biomechanical responses detected by a non-contact scheimpflug-based tonometer in eyes with glaucoma. Acta Ophthalmol. 2017;95(7):e556-e63. doi:10.1111/aos.13466

25. Lee R, Chang RT, Wong IY, Lai JS, Lee JW, Singh K. Novel parameter of corneal biomechanics that differentiate normals from glaucoma. $J$ Glaucoma. 2016;25(6):e603-9. doi:10.1097/ IJG.0000000000000284

26. Miki A, Yasukura Y, Weinreb RN, et al. Dynamic scheimpflug ocular biomechanical parameters in healthy and medically controlled glaucoma eyes. J Glaucoma. 2019;28(7):588-592. doi:10.1097/ IJG.0000000000001268

27. Pradhan ZS, Deshmukh S, Dixit S, et al. A comparison of the corneal biomechanics in pseudoexfoliation glaucoma, primary open-angle glaucoma and healthy controls using Corvis ST. PLoS One. 2020;15(10):e0241296. doi:10.1371/journal.pone.0241296

28. Fujishiro T, Matsuura M, Fujino Y, et al. The relationship between Corvis ST tonometry parameters and ocular response analyzer corneal hysteresis. J Glaucoma. 2020;29(6):479-484. doi:10.1097/ IJG.0000000000001486

29. Vinciguerra R, Elsheikh A, Roberts CJ, et al. Influence of pachymetry and intraocular pressure on dynamic corneal response parameters in healthy patients. J Refract Surg. 2016;32(8):550-561. doi:10.3928/ 1081597X-20160524-01

30. Roberts CJ, Mahmoud AM, Bons JP, et al. Introduction of two novel stiffness parameters and interpretation of air puff-induced biomechanical deformation parameters with a dynamic scheimpflug analyzer. $J$ Refract Surg. 2017;33(4):266-273. doi:10.3928/1081597X-20161221-03

31. Shah S, Laiquzzaman M, Mantry S, Cunliffe I. Ocular response analyser to assess hysteresis and corneal resistance factor in low tension, open angle glaucoma and ocular hypertension. Clin Exp Ophthalmol. 2008;36(6):508-513. doi:10.1111/j.1442-9071.2008.01 828.x
32. Murphy ML, Pokrovskaya O, Galligan M, O'Brien C. Corneal hysteresis in patients with glaucoma-like optic discs, ocular hypertension and glaucoma. BMC Ophthalmol. 2017;17(1):1. doi:10.1186/s12886016-0396-9

33. Vieira MJ, Pereira J, Castro M, Arruda H, Martins J, Sousa JP. Efficacy of corneal shape index in the evaluation of ocular hypertension, primary open-angle glaucoma and exfoliative glaucoma. Eur $J$ Ophthalmol. 2020;1120672120972020. doi:10.1177/112067212 0972020

34. Kimura A, Ando E, Fukushima M, et al. Secondary glaucoma in patients with familial amyloidotic polyneuropathy. Arch Ophthalmol. 2003;121(3):351-356. doi:10.1001/archopht.121.3.351

35. Beirao JM, Malheiro J, Lemos C, Beirao I, Costa P, Torres P. Ophthalmological manifestations in hereditary transthyretin (ATTR V30M) carriers: a review of 513 cases. Amyloid. 2015;22 (2):117-122. doi:10.3109/13506129.2015.1015678

36. Marta A, Vieira R, Figueiredo A, et al. Ahmed valve for secondary glaucoma in patients with hereditary transthyretin amyloidosis. Eye (Lond). 2021. doi:10.1038/s41433-021-01443-y

37. Latasiewicz M, Milla E, Giralt J, Molina JJ, Matas J. Nonpenetrating deep sclerectomy as an effective treatment of glaucoma related to familial amyloid polyneuropathy. J Glaucoma. 2015;24(5):e80-3. doi:10.1097/IJG.0000000000000126

38. Matsuura M, Hirasawa K, Murata H, Nakakura S, Kiuchi Y, Asaoka R. Using CorvisST tonometry to assess glaucoma progression. PLoS One. 2017;12(5):e0176380. doi:10.1371/journal. pone. 0176380

39. Ozkok A, Tamcelik N, Ozdamar A, Sarici AM, Cicik E. Corneal viscoelastic differences between pseudoexfoliative glaucoma and primary open-angle glaucoma. J Glaucoma. 2013;22(9):740-745. doi:10.1097/IJG.0000000000000036

40. Costin BR, Fleming GP, Weber PA, Mahmoud AM, Roberts CJ. Corneal biomechanical properties affect Goldmann applanation tonometry in primary open-angle glaucoma. J Glaucoma. 2014;23 (2):69-74. doi:10.1097/IJG.0b013e318269804b

41. Matsuura M, Murata H, Fujino Y, et al. Relationship between novel intraocular pressure measurement from Corvis ST and central corneal thickness and corneal hysteresis. Br J Ophthalmol. 2020;104 (4):563-568. doi:10.1136/bjophthalmol-2019-314370

42. Kniestedt C, Nee M, Stamper RL. Dynamic contour tonometry: a comparative study on human cadaver eyes. Arch Ophthalmol. 2004;122(9):1287-1293. doi:10.1001/archopht.122.9.1287

43. Kniestedt C, Nee M, Stamper RL. Accuracy of dynamic contour tonometry compared with applanation tonometry in human cadaver eyes of different hydration states. Graefes Arch Clin Exp Ophthalmol. 2005;243(4):359-366. doi:10.1007/s00417-004-1024-6

44. Feltgen N, Leifert D, Funk J. Correlation between central corneal thickness, applanation tonometry, and direct intracameral IOP readings. $\quad B r \quad J$ Ophthalmol. 2001;85(1):85-87. doi:10.1136/ bjo.85.1.85

45. Eliasy A, Chen KJ, Vinciguerra R, et al. Ex-vivo experimental validation of biomechanically-corrected intraocular pressure measurements on human eyes using the CorVis ST. Exp Eye Res. 2018;175:98-102. doi:10.1016/j.exer.2018.06.013

46. Wei Y, Xu L, Song H. Application of Corvis ST to evaluate the effect of femtosecond laser-assisted cataract surgery on corneal biomechanics. Exp Ther Med. 2017;14(2):1626-1632. doi:10.3892/ etm. 2017.4675

47. Seymenoglu G, Uzun O, Baser E. Surgically induced changes in corneal viscoelastic properties after 23-gauge pars plana vitrectomy using ocular response analyzer. Curr Eye Res. 2013;38(1):35-40. doi:10.3109/02713683.2012.707269

48. Boote C, Sigal IA, Grytz R, Hua Y, Nguyen TD, Girard MJA. Scleral structure and biomechanics. Prog Retin Eye Res. 2020;74:100773. doi:10.1016/j.preteyeres.2019.100773 


\section{Publish your work in this journal}

Clinical Ophthalmology is an international, peer-reviewed journal covering all subspecialties within ophthalmology. Key topics include Optometry; Visual science; Pharmacology and drug therapy in eye diseases; Basic Sciences; Primary and Secondary eye care; Patient Safety and Quality of Care Improvements. This journal is indexed on PubMed

Submit your manuscript here: https://www.dovepress.com/clinical-ophthalmology-journal
Central and CAS, and is the official journal of The Society of Clinical Ophthalmology (SCO). The manuscript management system is completely online and includes a very quick and fair peer-review system, which is all easy to use. Visit http://www.dovepress.com/ testimonials.php to read real quotes from published authors. 